\title{
Controlling Reactivity Through Liquid Assisted Grinding: The Curious Case of Mechanochemical Fluorination
}

\author{
Joseph L. Howard ${ }^{a}$, Yerbol Sagatov ${ }^{a}$, Laura Repusseau ${ }^{a}$, Christiane Schotten ${ }^{\mathrm{a}}$ and Duncan L.
} Browne ${ }^{\text {a* }}$

\begin{abstract}
We have identified an example of a mechanochemically milled organic reaction where liquid-assisted grinding controls the selectivity, such a phenomenon has not been reported/observed before. It was found that upon milling dibenzoylmethane with Selectfluor in the absence of any solvent, a 3:1 ratio of monofluorinated:difluorinated product was observed. Whereas, addition of $0.125 \mathrm{~mL}$ of acetonitrile ( $10 \%$ of the total volume of materials present) to the ground reaction mixture afforded 50:1 selectivity. Furthermore, this phenomenon is applicable to a small range of diketone substrates thus far explored. Additionally, we have demonstrated that difluorination can be achieved by simply switching from adding acetonitrile to addition of sodium carbonate. Most notably, in the latter case, is the reduced reaction time compared to a conventional solvent approach, 2 hours in the mill and $\mathbf{2 4}$ hours in the flask.
\end{abstract}

Mechanochemical milling methods represent an attractive process for the preparation of chemical products from a sustainability perspective. ${ }^{1}$ The concept of running reactions in the neat phase without solvent waste is irrefutably an important pursuit for a more sustainable future. ${ }^{2}$ Indeed, recent advances in the area of metal organic frameworks have demonstrated that such processes can be scaled to the manufacture level using a twin screw extrusion apparatus. ${ }^{3}$ Such equipment is already present in many industrial manufacturing plants for formulation, where reliable processing of powdered materials is necessary to meet regulatory demands. Most notably, this chemical processing tool has been found to vastly increase space time yields for MOF preparation. Still, solid-state milling, as applied to synthetic organic chemistry, is a relatively underexplored area given the potential gains that could be made against the economy and environment. In large part, we believe that the slow uptake of this technology is attributable to both a lack of understanding of the potential enabling attributes for organic synthesis and a poor understanding of how to optimize reactions (with respect to yield) through control of the operating parameters. ${ }^{1 c, 4}$ Liquid assisted grinding (LAG) represents one such phenomenon whereby the addition of small amounts of liquid can have a

a School of Chemistry, Main Building, Park Place, Cardiff University, Cardiff, CF10 3AT, UK.E-mail: dlbrowne@cardiff.ac.uk

Electronic Supplementary Information (ESI) available: Detailed experimental procedures and characterization data. See DOI: 10.1039/x0xx00000x

profound effect on the outcome of a milled reaction. Recently it was reported that both the quantity and nature of added liquid can result in the switching between polymorphs. ${ }^{5}$ Herein we describe a related observation concerning LAG for a synthetic fluorination reaction. Fluorinated molecules are in ever increasing demand due to their ability to dramatically enhance the properties of materials. ${ }^{6,7}$

Table 1. Optimization of conditions for the selective mono or difluorination of dibenzoylmethane under mechanochemical conditions.

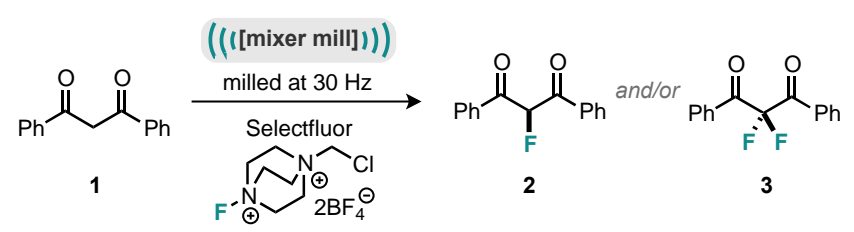

\begin{tabular}{|c|c|c|c|c|c|}
\hline Entry & $\begin{array}{l}\text { Selectfluor } \\
\text { (equiv) }\end{array}$ & $\begin{array}{l}\text { Time } \\
\text { (h) }\end{array}$ & Additive & $2[\%]^{[b]}$ & $3[\%]^{[b]}$ \\
\hline 1 & 1 & 1 & - & $53 \%$ & $4 \%$ \\
\hline 2 & 2 & 1 & - & $87 \%$ & $11 \%$ \\
\hline 3 & 2 & 0.5 & - & $53 \%$ & $4 \%$ \\
\hline 4 & 2 & 1 & $\mathrm{MeCN}(0.25 \mathrm{~mL})$ & $79 \%$ & $0 \%$ \\
\hline 5 & 2 & 2 & $\operatorname{MeCN}(0.25 \mathrm{~mL})$ & $91 \%$ & $7 \%$ \\
\hline 6 & 2 & 2 & - & $61 \%$ & $38 \%$ \\
\hline 7 & 2 & 2 & $\mathrm{H}_{2} \mathrm{O}(0.25 \mathrm{~mL})$ & $0 \%$ & $0 \%$ \\
\hline 8 & 2 & 2 & i-PrOH (0.25 mL) & $9 \%$ & $3 \%$ \\
\hline 9 & 2 & 2 & PhMe (0.25 mL) & $30 \%$ & $2 \%$ \\
\hline 10 & 2 & 2 & $\mathrm{CH}_{2} \mathrm{Cl}_{2}(0.25 \mathrm{~mL})$ & $20 \%$ & $0 \%$ \\
\hline 11 & 2 & 2 & $\operatorname{MeCN}(0.125 \mathrm{~mL})$ & $100 \%$ & $0 \%$ \\
\hline 12 & 2 & 2 & $\mathrm{Na}_{2} \mathrm{CO}_{3}$ (1 equiv) & $6 \%$ & $94 \%$ \\
\hline 13 & 2 & 2 & $\mathrm{~K}_{2} \mathrm{CO}_{3}$ (1 equiv) & $2 \%$ & $87 \%$ \\
\hline 14 & 2 & 2 & $\mathrm{Cs}_{2} \mathrm{CO}_{3}$ (1 equiv) & $2 \%$ & $68 \%$ \\
\hline 15 & 2 & 2 & $\mathrm{CaCO}_{3}$ (1 equiv) & $53 \%$ & $19 \%$ \\
\hline
\end{tabular}




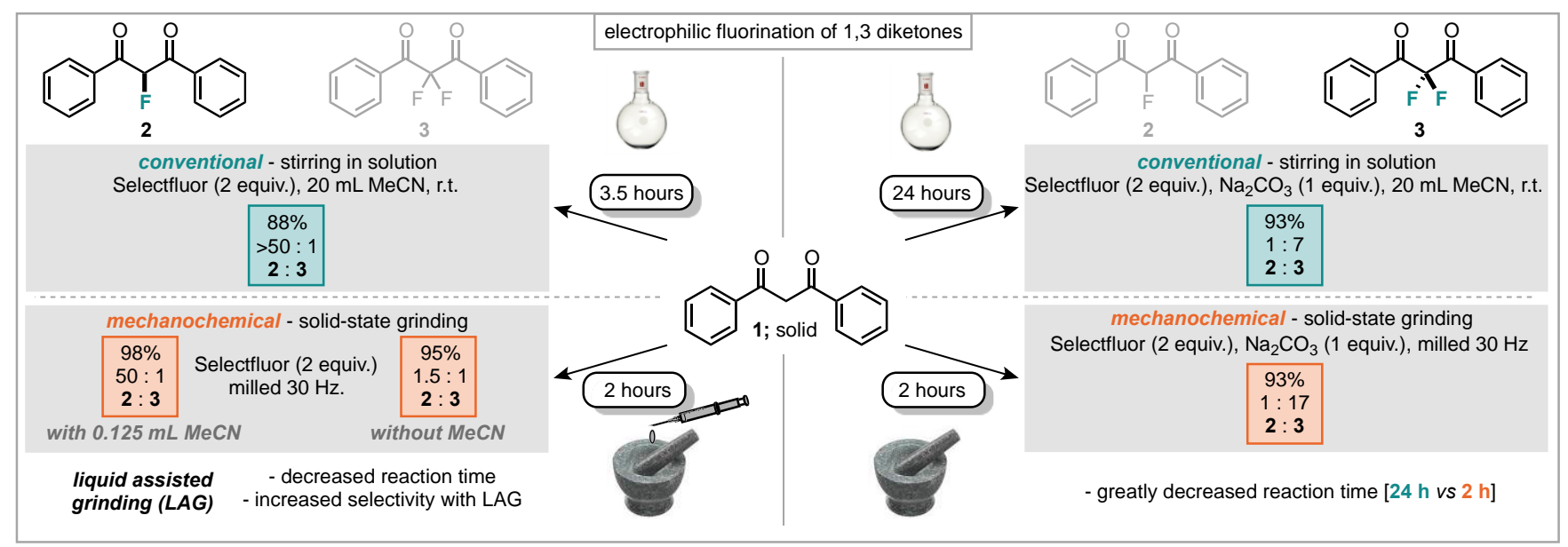

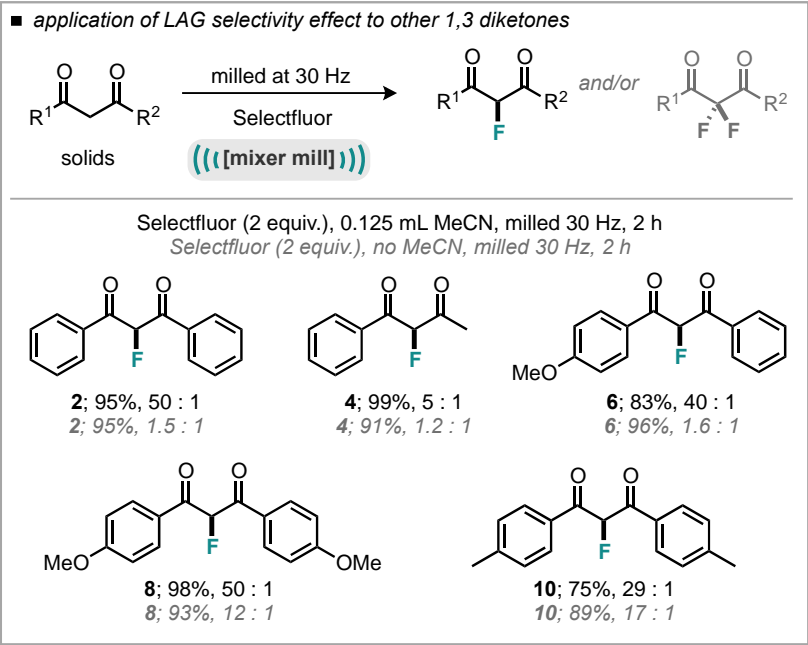

Figure 2. Scope of the enhanced selectivity effect afforded by Liquid Assisted Grinding of mechanochemical monofluorination reactions of 1,3 diketones.

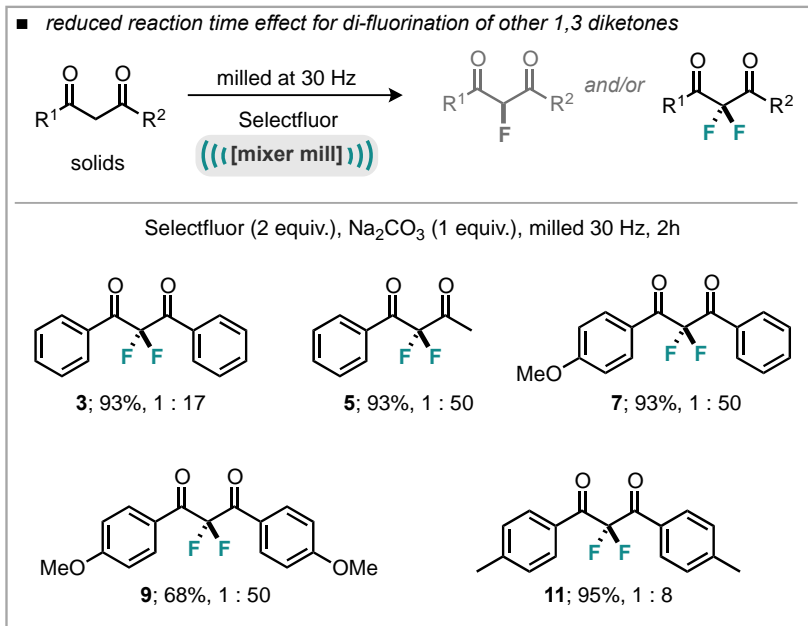

Figure 3. Scope of the reduced reaction time effect afforded by solid-state grinding of mechanochemical difluorination reactions of 1,3 diketones.
Figure 1. Summary of key mechanochemical observations and comparison to solvent based method. Isolated yields reported.

Given the prevalence of the carbon-fluorine bond in a range of important chemicals we were keen to investigate whether such a bond can be formed using solid-state milling techniques. Initial investigations commenced by treating solid dibenzoylmethane (1) with one equivalent of Selectfluor under mechanochemical mixer mill conditions. The reactions were performed on a $1 \mathrm{mmol}$ scale in $10 \mathrm{~mL}$ stainless steel jars with one stainless steel ball $(10 \mathrm{~mm}, 4.0$ g) and with a frequency of $30 \mathrm{~Hz}$ applied. Temperature is a difficult variable to control under milling conditions, but in all cases described here the jars were cool enough to handle immediately following the end of the grinding period, thus suggesting that the jar itself did not exceed $50^{\circ} \mathrm{C}$.

Pleasingly, after milling for 1 hour with equal reagent stoichiometries, a $53 \%$ yield of the monofluorinated product (2) was obtained (Table 1, Entry 1) with $4 \%$ of the difluorinated material (3) also present. The remaining mass balance was confirmed to be starting material (1). Initially increasing the equivalents of Selectfluor resulted in $87 \%$ of mono- and $11 \%$ difluorinated product (Table 1, Entry 2). Addition of a liquid, so called liquid assisted grinding or LAG, appeared to slow down the reaction, but most importantly, increased the selectivity towards the monofluorinated product (Table 1 Entry 4). ${ }^{8}$ Pushing the LAG reaction 
conditions further by increasing the time to two hours resulted in complete consumption of starting material with a 91:7 ratio of mono- to difluorinated products (Table 1 , Entry 5).

Neat milling under the same conditions resulted in inferior selectivity of $3: 2$, indicating that LAG is enabling improved selectivity. The nature of the liquid used for liquid assisted grinding was also explored, with isopropanol, toluene and dichloromethane all providing vastly inferior results (Table 1, Entries 8, 9 \& 10). Interestingly, added water seemingly resulted in a complete inhibition of the reaction, although notably Selectfluor is known to not be degraded by water (Table 1, Entry 7). ${ }^{9}$ Reducing the amount of added acetonitrile from $0.250 \mathrm{~mL}$ to $0.125 \mathrm{~mL}$ ( $10 \%$ of the total volume of all materials in the milling jar) resulted in further improvements to 100:0 (i.e. no difluorination). To put these results into perspective the solvent based reaction was also performed (in a similar fashion to Banks and co-workers), but with two equivalents of Selectfluor to make for a more accurate comparison. ${ }^{10}$ It was found that this method requires 3.5 hours to reach completion and afforded excellent selectivity (Figure 1). Also explored was the opportunity of proceeding directly to the difluorinated compound by simply adding base to the milled reaction. Addition of one equivalent of sodium carbonate provided an isolated yield of $90 \%$ of a 9:1 mixture of di- to monofluorinated products, again within 2 hours (Table 1 , Entry 12). Other carbonate bases proved less effective to mediate this transformation.

Figure 4. Summary of key mechanochemical observations and comparison to solvent based method for liquid substrates. Isolated yields reported. [a] $\mathrm{NaCl}$ used as a grinding agent/auxiliary/adsorbent for liquid reactants. The amount used is equal to twice that of the total of all other reactants.

When compared to the analogous solvent based reaction, the most notable observation was the reduced reaction time afforded by the mechanochemical technique, 24 hours against 2 hours (Figure 1 ).

In order to further probe these observations and see if they are more generally applicable, the monofluorination conditions with and without LAG for a small range of other 1,3 diketones were assessed (Figure 2). It was found, in all cases examined, that addition of $0.125 \mathrm{~mL}$ of acetonitrile to the solid reagents provided superior selectivity ratios to those without added acetonitrile, with all other variables remaining constant. In a similar manner, the generality of the observation that mechanochemical milling results in a reduced reaction time for the difluorination was also explored across the same range of substrates (Figure 3 ). Indeed, this was found to be true: milling this reaction results in significantly faster conversion to the desired product for all cases explored. Having assessed the reactivity of 1,3diketones with Selectfluor under mechanochemical conditions we turned to $\beta$-ketoesters which are both less reactive and liquid substrates. ${ }^{10}$ Milling of liquid/solid

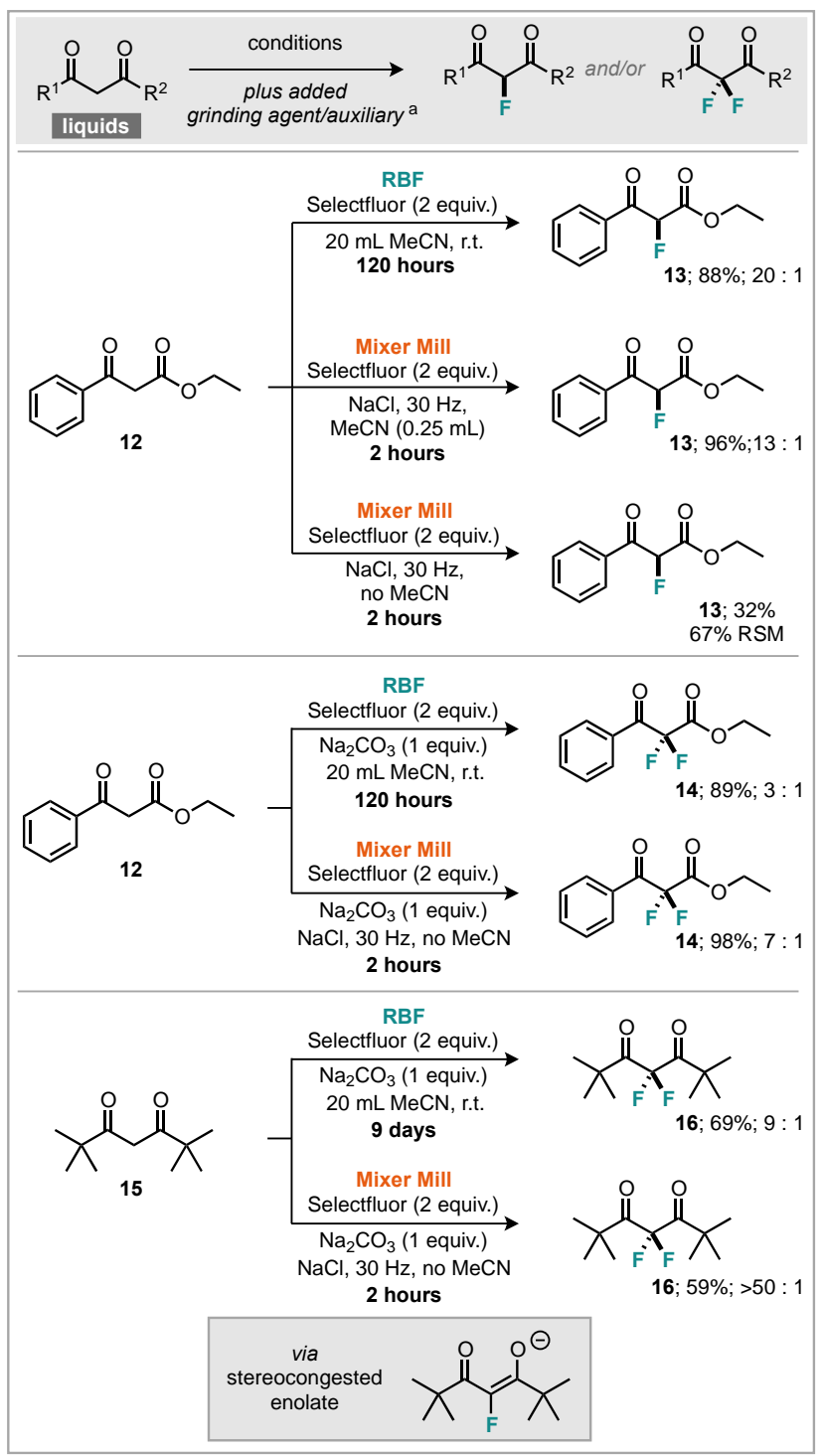

mixtures can result in the gumming of the reactor vessel and therefore poor mass transfer. Additionally, low boiling liquids can vaporize and release from the milling jars if appropriate care is not taken. To account for this, it is common to use an auxiliary material such as silica, alumina, talc or inorganic salts as a milling agent/auxiliary or adsorbent. ${ }^{11}$ In synthetic organic reactions some such materials could be considered far from innocent (such as the base used in Figure 3!). In pharmaceutical formulation science these materials are termed 'glidants' or 'lubricants' and assist in the uniform passage of powdered materials through screw extruders.12 On exploring the mechanochemical electrophilic fluorination of ethyl benzoylacetate, it was found that sodium chloride was a suitable material to permit adequate mass transfer and satisfactory results. Milling of the liquid ethyl benzoylacetate with Selectfluor and $\mathrm{NaCl}$ was explored in the presence and absence of LAG and compared to the traditional solvent based round bottom flask reaction (Figure 4). Most striking is the reduction in reaction time that is afforded by the solidstate milling approach (Figure 4 ). In this example the solvent based reaction occurs over 120 hours (5 days) whereas the 
milled reaction, with added acetonitrile, requires only two hours to run to completion! In the absence of (added) LAG the reaction mixture returned $67 \%$ recovered starting material and $32 \%$ yield of the monofluorination product. Difluorination of the liquid ethyl benzoylacetate was also possible within the same timeframe by adding sodium carbonate to the milled reaction mixture (in the absence of LAG), contrasting against the 5 day solvent-based reaction (Figure 4). With this newly found capability we finally explored the difluorination of 2,2,6,6-tetramethyl-3,5heptanedione, a liquid substrate whose intermediate monofluorinated compound features a highly stereocongested enolate.

In this instance the solvent based reaction conditions were found to require 9 days to proceed to $69 \%$ conversion (as measured by ${ }^{19} \mathrm{~F}$ NMR spectroscopy with an internal standard), whereas the mechanochemically milled reaction provided 59\% conversion in just two hours (Figure 4).

In conclusion, carbon-fluorine bond formation is possible under solid-state mechanochemical milling conditions. However, more significant is the observation that Liquid Assisted Grinding can be used to favor the formation of one reaction product over another and can give rise to improved selectivity. The precise effects of LAG on organic reactions are poorly characterized 8,13 and the exact rationale for the observed selectivity in this reaction remains unclear. ${ }^{14}$ Our current hypothesis is that it derives from changes in the crystalline form of the mono-fluorinated product, with such forms only accessible in the presence of added acetonitrile. These forms may be meta-stable nano-crystals as described by Belenguer, Hunter, Sanders and co-workers. ${ }^{5 a}$ Indeed, Jones and co-workers have described how different quantities of added LAG can lead to different polymorphs to that of a neat reaction. ${ }^{5 b}$ The latter point is a significant one as it implies that to understand such a phenomenon will require expertise in solid-state chemistry, organic reaction mechanism and mechano- or tribo-chemical methods. We believe the reaction manifold described herein is an ideal tool for such a study as the reaction is clean, can be monitored by ${ }^{19} \mathrm{~F}$ NMR and ceases to continue as soon as the product mixture is triturated away from the insoluble Selectfluor material. In addition, we have also demonstrated that mechanochemical milling can vastly reduce reaction times with little effect on yield and selectivity. This has been achieved with comparisons run in our laboratories with very closely related reaction conditions. This effect is applicable across both solid and liquid reagents, as long as the appropriate grinding agent is used.

\section{Acknowledgements}

D.L.B. thanks Cambridge Reactor Design for a Ph.D. award to J.L.H., The Bolashak International Scholarship of the President of the Republic of Kazakhstan for a Scholarship award to Y.S., the Erasmus programme for support of L.R. and the School of Chemistry at Cardiff University for generous support. We thank the EPSRC UK National Mass
Spectrometry Facility at Swansea University for mass spectrometry measurements.

\section{Notes and references}

1 For reviews about mechanochemistry and selected recent examples see (a) S. L. James, C. J. Adams, C. Bolm, D. Braga, P. Collier, T. Friščić, F. Grepioni, K. D. M. Harris, G. Hyett, W. Jones, A. Krebs, J. Mack, L. Maini, A. G. Orpen, I. P. Parkin, W. C. Shearouse, J. W. Steed and D. C. Waddell, Chem. Soc. Rev., 2012, 41, 413. (b) B. Rodríguez, A, Bruckmann, T. Rantanen and C. Bolm, Adv. Synth. Catal., 2007, 349, 2213. (c) A. Stolle, T. Szuppa, S. E. S. Leonhardt and B. Ondruschka, Chem. Soc. Rev., 2011, 40, 2317. (d) G.-W. Wang, Chem. Soc. Rev., 2013, 42, 7668. (e) D. Braga, L. Maini and F. Grepioni, Chem. Soc. Rev., 2013, 42, 7638. (f) E. Boldyreva, Chem. Soc. Rev., 2013, 42, 7719. (g) G. N. Hermann, P. Becker and C. Bolm, Angew. Chem. Int. Ed., 2016, 55, 3781. (h) S. Lou, Y. Mao, D.-Q. Xu, J. He, Q. Chen and Z. Xu, ACS Catal., 2016, 6, 3890. (i) Y. Zhou, F. Guo, C. E. Hughes,D. L. Browne, T. R. Peskett and K. D. M. Harris, Cryst. Growth Des., 2015, 15, 2901. (j) N. R. Rightmire, D. L. Bruns, T. P. Hanusa and W. W. Brennessel, Organometallics, 2016, 35, 1698.

2 (a) B. Ranu and A. Stolle Eds., Ball Milling Towards Green Synthesis; Royal Society Of Chemistry, Cambridge, U.K., 2014. (b) A. Sarkar, S. Santra, S. K. Kundu, A. Hajra, G. V. Zyryanov, O. N. Chupakhin, V. N. Charushin and A. Majee, Green Chem., 2016, 18, 4475. (c) R. A. Haley, A. R. Zellner, J. A. Krause, H. Guan and J. Mack, ACS Sustain. Chem. Eng., 2016, 4, 2464. (d) T. X. Métro, J. Bonnamour, T. Reidon, J. Sarpoulet, J. Martinez and F. Lamaty, Chem. Commun., 2012, 48, 11781. (e) T. X. Métro, J. Bonnamour, T. Reidon, A. Duprez, J. Sarpoulet, J. Martinez and F. Lamaty, Chem. Eur. J., 2015, 21, 12787. (f) E. Colacino, P. Nun, F. M. Colacino, J. Martinez and F. Lamaty, Tetrahedron, 2008, 64, 5569.

3 D. Crawford, J. Casaban, R. Haydon, N. Giri, T. McNally and S. L. James, Chem. Sci., 2015, 6, 1645.

4 (a) R. Trotzki, M. M. Hoffmann and B. Ondruschka, Green Chem., 2008, 10, 767. (b) G. Kaupp, CrystEngComm, 2011, 13, 3108. (c) T. Friščić, I. Halasz, P. J. Beldon, A. M. Belenguer, F. Adams, S. J. Kimber, V. Honkimäki and R. E. Dinnebier, Nat. Chem., 2013, 5, 66. (d) R. Schmidt, C. F. Burmeister, M. Balaz, A. Kwade and A. Stolle, Org. Process Res. Dev., 2015, 19, 427.

5 (a) A. M. Belenguer, G. I. Lampronti, A. J. Cruz-Cabeza, C.A. Hunter and J. K. M. Sanders, Chem. Sci., 2016, DOI: 10.1039/c6sc03457h. (b) D. Hasa, E. Miniussi, W. Jones, Cryst. Growth Des. 2016, 16, 4582.

6 (a) B. E. Smart, J. Fluor. Chem., 2001, 109, 3. (b) R. Filler and R. Saha, Future Med. Chem., 2009, 1, 777. (c) M. Morgenthaler, E. Schweizer, A. Hoffmann-Röder, F. Benini, R. E. Martin, G. Jaeschke, B. Wagner, H. Fischer, S. Bendels, D. Zimmerli, J. Schneider, F. Diederich, M. Kansy and K. Muller, ChemMedChem, 2007, 2, 1100. (d) D. B. Berkowitz and M. Bose, J. Fluor. Chem., 2001, 112, 13. (e) D. J. O'Hagan, J. Fluor. Chem., 2010, 131, 1071.

7 For reviews about organo-fluorine methods see $(a) \mathrm{J}$. Wang, M. Sánchez-Roselló, J. L. Aceña, C. del Pozo, A. E. Sorochinsky, S. Fustero, V. A. Soloshonok and H. Liu, Chem. Rev., 2014, 114, 2432. (b) T. Furuya, C. A. Kuttruff and T. Ritter, Curr. Opin. Drug Discov. Dev., 2008, 11, 803. (c) I. Hyohdoh, N. Furuichi, T. Aoki, Y. Itezono, H. Shirai, S. Ozawa, F. Watanabe, M. Matsushita, M. Sakaitani, P. S. Ho, K. Takanashi, N. Harada, Y. Tomii, K. 
Yoshinari, K. Ori, M. Tabo, Y. Aoki, N. Shimma, and H. likura, ACS Med. Chem. Lett., 2013, 4, 1059. (d) C. N. Neumann and T. Ritter, Angew. Chem. Int. Ed., 2015, 54, 3216. (e) J.-P. Bégué and D. Bonnet-Delpon, J. Fluor. Chem., 2006, 127, 992. (k) K. L. Kirk, Org. Process Res. Dev., 2008, 12, 305. (f) T. Liang,C. N. Neumann and T. Ritter, Angew. Chem. Int. Ed., 2013, 52, 8214. (m) J. A. Ma and D. Cahard, Chem. Rev., 2004, 104, 6119. (g) D. L. Browne and P. Richardson, Fluorination Approaches. In Synthetic Methods in Drug Discovery Volume 2, Royal Society of Chemistry, Cambridge, U.K., 2016, 263.

8 (a) T. Friščić, Chem. Soc. Rev., 2012, 41, 3493. (b) A. V. Trask, N. Shan, W. D. Motherwell, W. Jones, S. Feng, R. B. Tan and K. J. Carpenter, Chem. Commun. 2005, 7, 880. (c) T. Friščić, S. L. Childs, S. A. A. Rizvi and W. Jones, CrystEngComm., 2009, 11, 418.

9 G. Stavber, M. Zupan, M. Jereb and S. Stavber, Org. Lett., 2004, 6, 4973.

10 R. E. Banks, N. J. Lawrence and A. L. Popplewell, Chem. Commun., 1994, 3, 343.

11 (a) R. Schmidt, R. Thorwirth, T. Szuppa, A. Stolle, B. Ondruschka and H. Hopf, Chem. Eur. J., 2011, 17, 8129. (b) J. L. Do, C. Mottillo, D. Tan, V. Štrukil and T. Friščić, J. Am. Chem. Soc., 2015, 137, 2476. (c) R. Thorwirth, A. Stolle and B. Ondruschka, Green Chem., 2010, 12, 985.

12 J. Li and Y. Wu, Lubricants, 2014, 2, 21.

13 D. Tan, C. Mottillo, A. D. Katsenis, V. Štrukil and T. Friščic̈, Angew. Chem. Int. Ed., 2014, 53, 9321.

14 However, we have further probed this effect across different time points and have demonstrated that in this case LAG is causing improved selectivity, see supporting information for details. 